\title{
Synthesis and applications of new trans 1-indolyl-2-(1-methyl pyridinium and quinolinium-2-yl)ethylenes
}

\author{
Cosimo G. Fortuna, Vincenza Barresi, Nicola Musso, and Giuseppe Musumarra \\ Dipartimento di Scienze Chimiche, Università di Catania, Viale A. Doria, 695125 Catania, Italy \\ E-mail:cg.fortuna@unict.it
}

Dedicated to Professor Nicolò Vivona in the occasion of his $70^{\text {th }}$ anniversary

\begin{abstract}
The synthesis and spectroscopic characterization of trans 1-(indol-2-yl)-2-(1-methylpyridinium and 1-methylquinolinium-2-yl) ethylenes is reported. In vitro antitumour activity tests show that quinolinium derivatives are more active towards MCF7 (breast), LNCap (prostate) and U87 (human gliobastoma) carcinoma cells than pyridinium ones.
\end{abstract}

Keywords: Heterocycles, organic synthesis, citotoxicity, antitumour activity

\section{Introduction}

We have recently reported the design of new trans 2-(furan-2-yl)vinyl heteroaromatic iodides, ${ }^{1}$ with pyridinium, imidazolium and quinolinium as ethylene-linked heteroaromatic cations and halo substituted benzenes in the 5 position of the furan ring, with the aim to optimize the antitumour activities exhibited by imidazolium salts previously reported. ${ }^{2}$ In fact, previous work on the design, the synthesis and the antitumour activity of new trans 1-heteroaryl-2-(1,3dimethylimidazolium-2-yl) ethylenes ${ }^{2-5}$ confirmed the hypothesis that the presence of three aromatic moieties and of halogen atoms are the main structural features necessary to obtain satisfactory antiproliferative activities. On the basis of these results, we decided to introduce a new benzofused heteroaromatic ring such as indole in these compounds and to link pyridinium and quinolinium cations in the 2 position of the ethylene moiety in order to compare the activities of analogous derivatives possessing 3 and 4 aromatic rings. Here we report the synthesis and the biological evaluation of new trans 1-indolyl-2-(1-methylheteroar-2-yl) ethylenes against three type of tumour cells. 


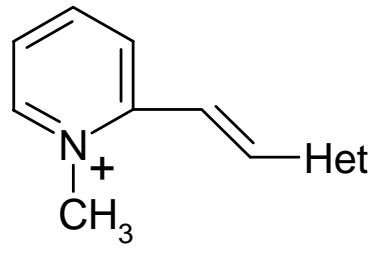

1-5

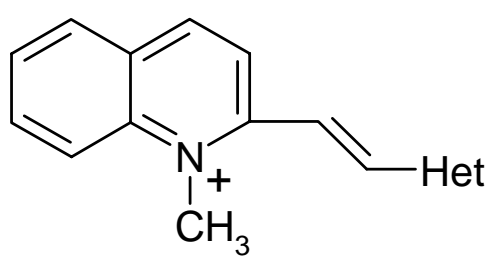

6-10

\begin{tabular}{ll}
\hline & Het \\
\hline $\mathbf{1 , 6}$ & Indol-5-yl \\
$\mathbf{2 , 7}$ & Indol-3-yl \\
$\mathbf{3 , 8}$ & 5-Br-indol-3-yl \\
$\mathbf{4 , 9}$ & 1-Me-indol-3-yl \\
$\mathbf{5 , 1 0}$ & Indol-4-yl \\
\hline
\end{tabular}

Compounds $1-10$, reported in Scheme 1, bearing heteroaromatic moieties with different electron donating abilities linked by a vinyl to the strong electron withdrawing pyridinium or quinolinium ring, belong to the class of the so called push-pull (donor-acceptor, D-A) molecules. In this context we here also report solvatochromic shifts due to the modulation of electronic parameters in both the donor and the acceptor heteroaromatic moieties.

\section{Results and Discussion}

The synthesis of trans 1-indolyl-2-(1-methylheteroar-2-yl) ethylenes 1-10 is straightforward and can be easily achieved by condensation of 1,2-dimethyl pyridinium iodide or 1,2-dimethyl quinolinium iodides with heteroaromatic aldehydes (see Experimental section). As expected, the pyridinium and quinolinium $\alpha$ methyls are quite reactive due to the strong electron withdrawing effect of the positively charged ring nitrogen.

Under appropriate conditions, outlined in the experimental section, pure trans isomers $\mathbf{1}-\mathbf{1 0}$ were obtained, as evidenced by the ethylenic protons J coupling constants in the NMR spectra (see Experimental section). Compounds 1 - 10 possess electron donating moieties linked by a vinyl linker to the pyridinium or quinolinium ring. Derivatives with extended conjugation are expected to exhibit significant solvatochromic shifts. The absorption maxima of cations $\mathbf{1}-\mathbf{1 0}$ in protic and aprotic solvents of various polarity are reported in Table 1. In particular we report for cations 4 and 8 - 10 the absorption maxima in 4 solvents at different polarity, and for other compounds the absorption maxima in 3 solvents due to solubility problems in chloroform. In the last column, we reported the shift of $\lambda$ of the max absorption between a non polar solvent or less polar solvent and a polar one. Compounds exhibiting significant solvatochromic shifts in Table 1, will be characterized by Electric-Field-Induced Second Harmonic Generation (EFISH) and Hyper-Rayleigh experiments in order to envisage possible future applications in NLO. 
Table 1. Absorption maxima for 1- indolyl-2-(1-methylheteroar-2-yl) ethylenes 1-10 in different solvents

\begin{tabular}{lcccccc}
\hline $\begin{array}{l}\text { Compounds } \backslash \\
\text { Solvents }\end{array}$ & $\mathrm{CHCl}_{3}$ & $\mathrm{EtOH}$ & $\mathrm{MeOH}$ & $\mathrm{H}_{2} \mathrm{O}$ & $\begin{array}{c}\Delta \lambda_{\max }(\mathrm{nm}) \\
\left(\mathrm{CHCl}_{3}-\mathrm{H}_{2} \mathrm{O}\right)\end{array}$ & $\begin{array}{c}\Delta \lambda_{\max }(\mathrm{nm}) \\
\left(\mathrm{EtOH}-\mathrm{H}_{2} \mathrm{O}\right)\end{array}$ \\
\hline $\mathbf{1}$ & - & 400 & 380.35 & 378.34 & & 21.66 \\
$\mathbf{2}$ & - & 430.73 & 429.72 & 408.56 & & 22.17 \\
$\mathbf{3}$ & - & 479.60 & 419.14 & 414.61 & & 64.99 \\
$\mathbf{4}$ & 443.83 & 431.74 & 430.73 & 417.63 & 26.2 & \\
$\mathbf{5}$ & - & 423.68 & 419.65 & 398.48 & & 25.2 \\
$\mathbf{6}$ & - & 448.87 & 439.80 & 472.04 & & 23.17 \\
$\mathbf{7}$ & - & 480.10 & 473.05 & 449.87 & & 30.23 \\
$\mathbf{8}$ & 509.29 & 470.03 & 463.98 & 440.81 & 68.48 & \\
$\mathbf{9}$ & 505.29 & 487.15 & 483.12 & 467.00 & 38 & \\
$\mathbf{1 0}$ & 473.05 & 464.99 & 464.99 & 438.79 & 34.26 & \\
\hline
\end{tabular}

However, the major purpose of the present work is to test the in vitro antitumour activity of the title water soluble trans 1-indolyl-2-(1-methylheteroar-2-yl)ethylenes and to relate the results with those of the previous studies published from our group.

The in vitro antitumour activity of the water soluble compounds synthesized in the present work was then tested against three tumour cell lines, breast carcinoma (MCF7), prostate carcinoma (LNCap) and human gliobastoma (U87). The in vitro activities, expressed as log $\mathrm{GI}_{50}$ values (see Experimental Section), are recorded in Table 2, together with that of 2,6-di-[2-(furan2-yl)vinyl]pyridinium iodide $\left(\mathrm{PF}_{2}\right)^{6}$, the most active compounds in previous in vitro tests, also reported for comparisons. It is worth mentioning that, in order to obtain comparable biological tests, $\log \mathrm{GI}_{50}$ in Table 2 were all measured in the same experiment. The percent of growth and the inhibition exerted by different doses $(0.01-100 \mu \mathrm{M})$ are recorded in Figure 1. In addition to the antiproliferative effects $\left(\log \mathrm{GI}_{50}\right)$, two derivatives exhibit a significant cytotoxic activity, expressed as $\log \mathrm{LC}_{50}$ values and reported in Table 3. 

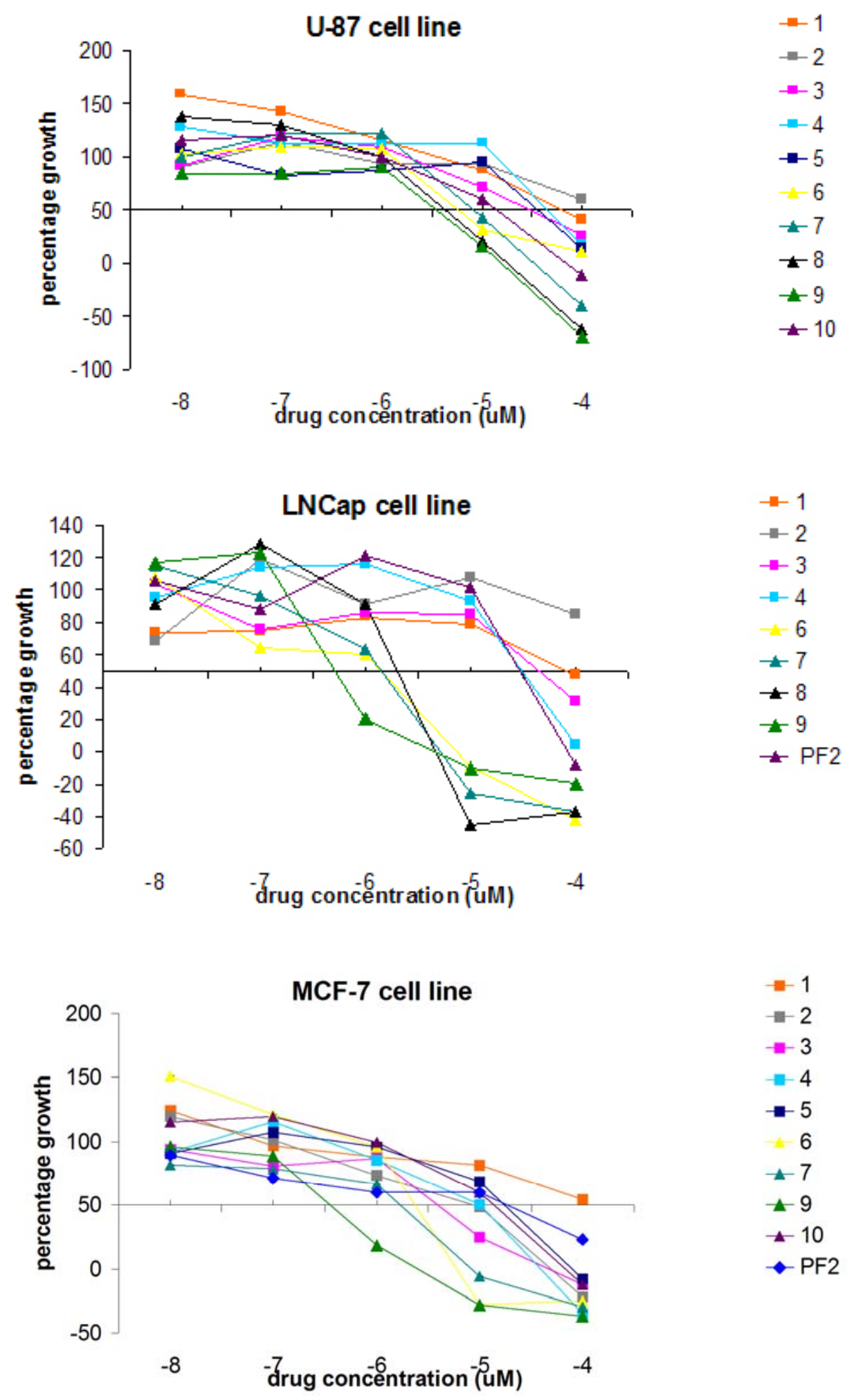

Figure 1. Dose - response curves of the antiproliferative activities of synthesised compounds and PF2 on U87, LNCap and MCF7 cell lines. 
Table 2. In vitro antitumor activities, expressed as $\log \mathrm{GI}_{50}$, for U87, LNCap and MCF7 cell lines

\begin{tabular}{cccccccccccc}
\hline $\begin{array}{c}\text { Cmpds/ } \\
\text { Cell line }\end{array}$ & 1 & 2 & 3 & 4 & 5 & 6 & 7 & 8 & 9 & 10 & $\mathrm{PF}_{2}$ \\
\hline U87 & -4.20 & $>-4.00$ & -4.50 & -4.37 & -4.45 & -5.40 & -5.14 & -5.42 & -5.48 & -4.47 & - \\
LNCap & -4.00 & $>-4.00$ & -4.25 & -4.50 & - & -5.80 & -5.80 & -5.72 & -6.48 & - & -4.50 \\
MCF-7 & $>-4.00$ & -5.00 & -5.40 & -5.00 & -4.70 & -5.60 & -5.76 & - & -6.38 & -5.00 & -4.70 \\
\hline
\end{tabular}

Table 3. In vitro citotoxicity, expressed as $\log \mathrm{LC}_{50}$, for U87, LNCap and MCF7 cell lines

\begin{tabular}{lcc}
\hline Cmpds/Cell line & 8 & 9 \\
\hline U87 & -4.00 & -4.26 \\
LNCap & -5.10 & - \\
\hline
\end{tabular}

Compounds 6 and 7 in Table 2 exhibit activity values slightly better than that of $\mathrm{PF}_{2}$, while compound 9 presents the highest value recorded in Table 2, towards all three cell lines.

\section{Conclusions}

New trans 1-indolyl-2-(1-methylquinolinium-2-yl) ethylene iodides tested in the present study are more active than 1,2 diheteroaryl ethylenes previously reported ${ }^{1,2,6,7}$ suggesting that the presence of four aromatic moieties increase the antitumour activities. Moreover, the significant solvatochromic shifts exhibited by these new push-pull derivatives indicate them as promising candidates for further investigations aimed at non linear optics applications.

\section{Experimental Section}

General Procedures. Heteroaromatic carboxaldehydes, Aldrich commercial products, were used without further purification. UV-Vis difference spectra were recorded on a Perkin Elmer Lambda $2 \mathrm{~S}$ spectrometer. $1 \mathrm{H}$ NMR spectra were recorded on a Varian Unity Inova spectrometer operating at $500 \mathrm{MHz}$, at $25^{\circ} \mathrm{C}$ in $\left(\mathrm{CD}_{3}\right)_{2} \mathrm{SO}$ using TMS as internal standards. The spectral width was set to $5,000 \mathrm{~Hz}$, with an excitation pulse of 60 degrees, an acquisition time of $3.5 \mathrm{~s}$ and a digital resolution after zero-filling of $0.15 \mathrm{~Hz} / \mathrm{pt}$. Fast Atom Bombardment (FAB) mass spectra were recorded on a double focusing Kratos MS 500 mass spectrometer equipped with the standard FAB source. Mass spectra ESI were recorded on a LCQ Deca Finnigan system with a flow of $5 \mu \mathrm{l} / \mathrm{min}$. Compounds 1-10, all iodide salts, were obtained by refluxing in ethanol equimolar amounts of 1,2- dimethylpicolinium iodide or 1,2- dimethyl quinolinium iodide and 
the appropriate indole aldehyde in the presence of few drops of base. The resulting precipitate was recrystallized from ethanol.

Details on the synthetic conditions and products characterization are reported below:

1-(Indol-5-yl)-2-(1-methylpiridium-2-yl) ethylene iodide (1). 20\% $\mathrm{NaOH}$, EtOH, 3hrs, reflux. Yields: $17 \%$; (green prisms from ethanol); $\mathrm{mp} 244-245^{\circ} \mathrm{C}$;

${ }^{1} \mathrm{H}$ NMR , $\left(\right.$ DMSO-d $\left._{6}\right), \delta=11.41 \mathrm{NH}(1 \mathrm{H}, \mathrm{s}) ; 8.81 \mathrm{H}_{6}(1 \mathrm{H}, \mathrm{d}, \mathrm{J}=6 \mathrm{~Hz}) ; 8.51 \mathrm{H}_{3}(1 \mathrm{H}, \mathrm{d}, \mathrm{J}=8$ $\mathrm{Hz}) ; 8.41 \mathrm{H}_{4}(1 \mathrm{H}, \mathrm{t}, \mathrm{J}=8.5 \mathrm{~Hz}) ; 8.05 \mathrm{H}_{4}$, $\mathrm{Ha}(2 \mathrm{H}, \mathrm{d}, \mathrm{J}=16 \mathrm{~Hz}) ; 7.80 \mathrm{H}_{5}(1 \mathrm{H}, \mathrm{t}, \mathrm{J}=7 \mathrm{~Hz}) ; 7.67$ H6' $(1 \mathrm{H}, \mathrm{d}, \mathrm{J}=8.5 \mathrm{~Hz}) ; 7.51 \mathrm{Hb}(1 \mathrm{H}, \mathrm{d}, \mathrm{J}=15 \mathrm{~Hz}) ; 7.49 \mathrm{H} 7$ ' $(1 \mathrm{H}, \mathrm{d}, \mathrm{J}=8 \mathrm{~Hz}) ; 7.45 \mathrm{H} 3$ '(1H, d, J $=7 \mathrm{~Hz}) ; 6.54 \mathrm{H} 2$ ' $(1 \mathrm{H}, \mathrm{s}) ; 4.35 \mathrm{H} 1(3 \mathrm{H}, \mathrm{s})$. MS : positive ESI $\left(\mathrm{M}^{+} 235.3\right)$

1-(Indol-3-yl)-2-(1-methylpiridium-2-yl) ethylene iodide (2). 20\% $\mathrm{NaOH}$, EtOH, 5hrs, reflux. Yields: $100 \%$; (green prisms from ethanol); $\mathrm{mp} 265-266^{\circ} \mathrm{C}$;

${ }^{1} \mathrm{H}$ NMR $,\left(\mathrm{DMSO}_{\mathrm{d}}\right), \delta=8.73 \mathrm{H6}(1 \mathrm{H}, \mathrm{d}, \mathrm{J}=6 \mathrm{~Hz}) ; 8.48 \mathrm{H} 3(1 \mathrm{H}, \mathrm{d}, \mathrm{J}=8 \mathrm{~Hz}) ; 8.34 \mathrm{H} 4(1 \mathrm{H}, \mathrm{t}$, $\mathrm{J}=8 \mathrm{~Hz}), 8.24 \mathrm{Ha}(1 \mathrm{H}, \mathrm{d}, \mathrm{J}=15.5 \mathrm{~Hz}) ; 8.11 \mathrm{H} 2^{\prime}, \mathrm{H} 7$ ' $(2 \mathrm{H}, \mathrm{t}, \mathrm{J}=5.75 \mathrm{~Hz}) ; 7.67 \mathrm{H} 5$ (1H , t, J = $6.75 \mathrm{~Hz}) ; 7.52 \mathrm{H} 4$ ' (1H, d, J = $7 \mathrm{~Hz}) ; 7.26 \mathrm{H} 5^{\prime}, \mathrm{H}$ 6', Hb $(3 \mathrm{H}, \mathrm{m}, \mathrm{J}=16 \mathrm{~Hz}), 4.32 \mathrm{H} 1$ (3H, s); $11,41 \mathrm{NH}(1 \mathrm{H}, \mathrm{s}) . \mathrm{MS}$ : positive ESI $\left(\mathrm{M}^{+} 235.3\right)$

1-(5-Br-indol-3-yl)-2-(1-methylpiridium-2-yl) ethylene iodide (3). 20\% $\mathrm{NaOH}, \mathrm{EtOH}, 4 \mathrm{hrs}$, reflux. Yields: $11 \%$; (green needles from ethanol); $\mathrm{mp} 250-251^{\circ} \mathrm{C}$;

${ }^{1} \mathrm{H} \mathrm{NMR},\left(\mathrm{DMSO}_{-} \mathrm{d}_{6}\right), \delta=12.16 \mathrm{NH}(1 \mathrm{H}, \mathrm{s}) ; 8.75 \mathrm{H6}(1 \mathrm{H}, \mathrm{d}, \mathrm{J}=6.5 \mathrm{~Hz}) ; 8.52 \mathrm{H} 3(1 \mathrm{H}, \mathrm{d}, \mathrm{J}=8.5$ $\mathrm{Hz})$; $8.37 \mathrm{H} 4(1 \mathrm{H}, \mathrm{t}) ; 8.28 \mathrm{Ha}, \mathrm{H} 4$ '(2H, t, J = 16Hz); $7.72 \mathrm{H} 5$ (1H, t, J = 6.5Hz); $7.48 \mathrm{H6}$ ' (1H, d, $\mathrm{J}=8 \mathrm{~Hz}) ; 7.37 \mathrm{H} 2^{\prime}, \mathrm{H}^{\prime}$ '(2H , m, J = 9 Hz), $7.27 \mathrm{Hb}(1 \mathrm{H}, \mathrm{d}, \mathrm{J}=15 \mathrm{~Hz}), 4.32 \mathrm{H1}(3 \mathrm{H}, \mathrm{s}) . \mathrm{MS}$ : positive ESI $\left(\mathrm{M}^{+} 313.2\right)$

1-(1-Methyl-indol-3-yl)-2-(1-methylpiridium-2-yl) ethylene iodide (4). $20 \% \mathrm{NaOH}, \mathrm{EtOH}$, 2hrs, reflux. Yields: 6\%; (orange needles from ethanol); mp 276-277 ${ }^{\circ} \mathrm{C}$;

${ }^{1} \mathrm{H}$ NMR , (DMSO-d $), \delta=8.74 \mathrm{H6}(1 \mathrm{H}, \mathrm{d}, \mathrm{J}=6 \mathrm{~Hz}) ; 8.51 \mathrm{H} 3(1 \mathrm{H}, \mathrm{d}, \mathrm{J}=8.5 \mathrm{~Hz}) ; 8.34 \mathrm{H} 4(1 \mathrm{H}, \mathrm{t}$, $\mathrm{J}=8.25 \mathrm{~Hz}) ; 8.24 \mathrm{Ha}(1 \mathrm{H}, \mathrm{d}, \mathrm{J}=16 \mathrm{~Hz}), 8.11 \mathrm{H} \mathrm{2}$ ', H7' $(2 \mathrm{H}, \mathrm{m}) ; 7.68 \mathrm{H} 5$ (1H, t, J = $6.75 \mathrm{~Hz})$; 7.59 H4' (1H, d, J = 8.5 Hz); 7.31 H5' H6' (2 H, m); 7.22 Hb (1H, d, J = 16Hz); $3.32 \mathrm{H1}(3 \mathrm{H}, \mathrm{s})$; $3.31 \mathrm{H} 1$ ' $(3 \mathrm{H}, \mathrm{s})$. MS: positive ESI $\left(\mathrm{M}^{+} 249.3\right)$

1-(Indol-4-yl)-2-(1-methylpiridium-2-yl) ethylene iodide (5). Piperidine, EtOH, 30 minutes, reflux. Yields: 81\%; (Yellow needles from ethanol); mp 243-244 ${ }^{\circ} \mathrm{C} ;{ }^{1} \mathrm{H}$ NMR , (DMSO-d 6 ), $\delta=$ $11.56 \mathrm{NH}(1 \mathrm{H}, \mathrm{s}) ; 8.88 \mathrm{H} 3(1 \mathrm{H}, \mathrm{d}, \mathrm{J}=5.5 \mathrm{~Hz}) ; 8.68 \mathrm{H6}(1 \mathrm{H}, \mathrm{d}, \mathrm{J}=8 \mathrm{~Hz}) ; 8.48 \mathrm{H} 5(1 \mathrm{H}, \mathrm{t}, \mathrm{J}=8$ $\mathrm{Hz}), 8.27 \mathrm{Ha}(1 \mathrm{H}, \mathrm{d}, \mathrm{J}=16 \mathrm{~Hz}) ; 7.88 \mathrm{H} 4(1 \mathrm{H}, \mathrm{t}, \mathrm{J}=6.75 \mathrm{~Hz}) ; 7.62 \mathrm{H6}{ }^{\prime}(1 \mathrm{H}, \mathrm{t}, \mathrm{J}=5.75 \mathrm{~Hz})$, 7.57 H 5'- H7' (2H, m ); $7.23 \mathrm{Hb}-\mathrm{H}$ 3' (2H, t, J = 15.5 Hz), 7. 02 H2' (1H, d ); $3.32 \mathrm{H} 1$ (3H, s).. MS: positive ESI $\left(\mathrm{M}^{+} 249.3\right)$

1-(Indol-5-yl)-2-(1-methylquinolinium-2-yl) ethylene iodide (6). Pyperidine, EtOH, 4 hrs, reflux. Yields: 100\%; (Red needles from ethanol); mp 243-245 ${ }^{\circ} \mathrm{C} ;{ }^{1} \mathrm{H}$ NMR , (DMSO-d 6$), \delta=$ $8.96 \mathrm{H} 4(1 \mathrm{H}, \mathrm{d}, \mathrm{J}=9 \mathrm{~Hz}) ; 8.59 \mathrm{H} 3(1 \mathrm{H}, \mathrm{d}, \mathrm{J}=9.5 \mathrm{~Hz}) ; 8.51 \mathrm{H} 8(1 \mathrm{H}, \mathrm{d}, \mathrm{J}=9 \mathrm{~Hz}) ; 8.40 \mathrm{Ha}(1 \mathrm{H}$, $\mathrm{d}, \mathrm{J}=15.5 \mathrm{~Hz}) ; 8.31 \mathrm{H} 5(1 \mathrm{H}, \mathrm{d}, \mathrm{J}=8 \mathrm{~Hz}) ; 8.21 \mathrm{H} 4{ }^{\prime}(1 \mathrm{H}, \mathrm{s}) ; 8.14 \mathrm{H6}(1 \mathrm{H}, \mathrm{d}, \mathrm{J}=8 \mathrm{~Hz}) ; 7.91 \mathrm{H} 7$ $(1 \mathrm{H}, \mathrm{t}, \mathrm{J}=7.25 \mathrm{~Hz}) ; 7.82 \mathrm{Hb}(1 \mathrm{H}, \mathrm{d}, \mathrm{J}=16 \mathrm{~Hz}) ; 7.81 \mathrm{H6}$ ' (1H, d, J = 7.5 Hz); $7.54 \mathrm{H} 7$ ' (1H, d, J 
$=8.5 \mathrm{~Hz}) ; 7.47 \mathrm{H} 3^{\prime}(1 \mathrm{H}, \mathrm{d}, \mathrm{J}=8.5 \mathrm{~Hz}) ; 6.59 \mathrm{H} 2$ ' $(1 \mathrm{H}, \mathrm{s}) ; 11.44 \mathrm{NH}(1 \mathrm{H}, \mathrm{s}) ; 4.54 \mathrm{H} 1(3 \mathrm{H}, \mathrm{s})$. MS: positive ESI $\left(\mathrm{M}^{+} 285.3\right)$

1-(Indol-3-yl)-2-(1-methylquinolinium-2-yl) ethylene iodide (7). Piperidine, EtOH, 6 hrs, reflux. Yields: 50\%; (green needles from ethanol); mp $253-255^{\circ} \mathrm{C}$; ${ }^{1} \mathrm{H}$ NMR , (DMSO- $\mathrm{d}_{6}$ ), $\delta=12.34 \mathrm{NH}(1 \mathrm{H}, \mathrm{s}) ; 8.80 \mathrm{H} 4(1 \mathrm{H}, \mathrm{d}, \mathrm{J}=9.5 \mathrm{~Hz}) ; 8.60 \mathrm{Ha}(1 \mathrm{H}, \mathrm{d}, \mathrm{J}=15.5 \mathrm{~Hz}), 8.56 \mathrm{H} 3(1 \mathrm{H}, \mathrm{d}, \mathrm{J}$ $=9.5 \mathrm{~Hz}) ; 8.42 \mathrm{H} 8(1 \mathrm{H}, \mathrm{d}, \mathrm{J}=8.5 \mathrm{~Hz}) ; 8.26 \mathrm{H} 5(1 \mathrm{H}, \mathrm{t}, \mathrm{J}=8 \mathrm{~Hz}) ; 8.19 \mathrm{H} 5$ ' (1H, t, J = 4.25 Hz); $8.1 \mathrm{H6}(1 \mathrm{H}, \mathrm{t}, \mathrm{J}=7.75 \mathrm{~Hz}) ; 7.83 \mathrm{H} 7(1 \mathrm{H}, \mathrm{t}, \mathrm{J}=7.5 \mathrm{~Hz}) ; 7.56 \mathrm{Hb}, \mathrm{H} 7$ ' $(2 \mathrm{H}, \mathrm{t}, \mathrm{J}=16 \mathrm{~Hz}) ; 7.30$ H5', H6' $(2 \mathrm{H}, \mathrm{t}, \mathrm{J}=4.25 \mathrm{~Hz}) ; 4.46 \mathrm{H} 4$ ' $(1 \mathrm{H}, \mathrm{d}, \mathrm{J}=4.25 \mathrm{~Hz}) ; 4.40 \mathrm{H} 1$ (3H, s). MS: positive ESI $\left(\mathrm{M}^{+} 285.4\right)$

1-(5-Br-indol-3-yl)-2-(1-methylquinolinium-2-yl) ethylene iodide (8). 20\% $\mathrm{NaOH}, \mathrm{EtOH}, 24$ hrs, reflux. Yields: 65\%; (orange needles from ethanol); mp 207-208 ${ }^{\circ}$; ${ }^{1} \mathrm{H}$ NMR , (DMSO-d 6 ), $\delta=8.42 \mathrm{NH}(1 \mathrm{H}, \mathrm{s}) ; 8.86 \mathrm{H} 4(1 \mathrm{H}, \mathrm{d}, \mathrm{J}=9 \mathrm{~Hz}) ; 8.66 \mathrm{Ha}(1 \mathrm{H}, \mathrm{d}, \mathrm{J}=16 \mathrm{~Hz}), 8.63 \mathrm{H} 2$ ' (1H, d, J = $8 \mathrm{~Hz})$; $8.51 \mathrm{H} 4$ ' (1H, s) $8.46 \mathrm{H} 5 \mathrm{H} 8(2 \mathrm{H}, \mathrm{m}) ; 8.26 \mathrm{H6}$ '(1H, d, J = 7.5 Hz); $8.10 \mathrm{H6}(1 \mathrm{H}, \mathrm{t}, \mathrm{J}=8$ $\mathrm{Hz}) ; 7.86 \mathrm{H} 7(1 \mathrm{H}, \mathrm{t}, \mathrm{J}=7.5 \mathrm{~Hz}) ; 7.63 \mathrm{H} \mathrm{b}(1 \mathrm{H}, \mathrm{d}, \mathrm{J}=15 \mathrm{~Hz}) ; 7.51 \mathrm{H} 3(1 \mathrm{H}, \mathrm{d}, \mathrm{J}=9 \mathrm{~Hz}) ; 7.41$ H7'(1H, d, J = 8.5 Hz); 4.38 H1 (3H, s) MS: positive ESI $\left(\mathrm{M}^{+} 363.3, \mathrm{M}^{+} 2\right.$ 365.2)

1-(1-Methyl-indol-3-yl)-2-(1-methylquinolinium-2-yl) ethylene iodide (9). Piperidine, EtOH, $1 \mathrm{hrs}$, reflux. Yields: 27\%; (violet needles from ethanol); mp $188-190{ }^{\circ} \mathrm{C} ;{ }^{1} \mathrm{H} \mathrm{NMR}$, (DMSO-d 6 ), $\delta=8.79 \mathrm{H} 4(1 \mathrm{H}, \mathrm{d}, \mathrm{J}=9.5 \mathrm{~Hz}) ; 8.57 \mathrm{Ha}-\mathrm{H} 3(2 \mathrm{H}, \mathrm{d}, \mathrm{J}=16 \mathrm{~Hz}) ; 8.40 \mathrm{H} 8(1 \mathrm{H}, \mathrm{d}, \mathrm{J}=9 \mathrm{~Hz}) ; 8.33$ H2' (1H, s); 8.22 H5 - H5' (1H, t, J = 7.75 Hz); 8.07 H6 (1H, t, J = 8 Hz); $7.82 \mathrm{H} 7$ (1H, t, J = 7.5 $\mathrm{Hz}) ; 7.62 \mathrm{H7}$ ' (1H, d, J = 7.5 Hz); 7.52 Hb (1H, d, J = 15,5 Hz); 7.36 H6' (2H, t, J = 7.25 Hz); $4.62 \mathrm{H} 1(3 \mathrm{H}, \mathrm{s}) 4.61 \mathrm{H} 1$ ' $(3 \mathrm{H}, \mathrm{s})$. MS: positive ESI $\left(\mathrm{M}^{+} 299.3\right)$

1-(Indol-4-yl)-2-(1-methylquinolinium-2-yl) ethylene iodide (10). Pyperidine, EtOH, 5 hrs, reflux. Yields: 82\%; (red needles from ethanol); mp 221-222 ${ }^{\circ} \mathrm{C} ;{ }^{1} \mathrm{H}$ NMR , $\left(\mathrm{DMSO}-\mathrm{d}_{6}\right), \delta=9.04$ $\mathrm{NH}(1 \mathrm{H}, \mathrm{d}, \mathrm{J}=8.5 \mathrm{~Hz}) ; 8.76 \mathrm{H3}(1 \mathrm{H}, \mathrm{d}, \mathrm{J}=9 \mathrm{~Hz}), 8.57 \mathrm{H} 8-\mathrm{Ha}(2 \mathrm{H}, \mathrm{m}) ; 8.34 \mathrm{H} 5$ (1H, d, J = 8 $\mathrm{Hz}) ; 8.18 \mathrm{H6}(1 \mathrm{H}, \mathrm{t}, \mathrm{J}=7.75 \mathrm{~Hz}) ; 7.95 \mathrm{H} 4-\mathrm{H} 7$ (2H, m); 7.81 H5' (1H, d, J = 7.5 Hz), $7.62 \mathrm{H7}$ ' - H3'(2H, m); 7.27 H6'-Hb (2H, dd, J = 15.5Hz) ; $7.12 \mathrm{H} 2$ ' $(1 \mathrm{H}, \mathrm{s}) 4.58 \mathrm{H} 1$ (3H, s). MS: positive ESI $\left(\mathrm{M}^{+} 285.4\right)$.

\section{Biological essays}

Human cell lines (LNCap, MCF7, U87). Human prostate adenocarcinoma cells (LNCap) and human gliobastoma cells (U87) were grown in RPMI 1640. Human mammary adenocarcinoma (MCF7) were grown in Dulbecco's MEM (DMEM), $1.0 \mathrm{~g} / 1 \mathrm{D}$-glucose. Each medium was supplemented with $10 \%$ (vol/vol) heat-inactivated fetal bovine serum, $2 \mathrm{mM}$ L-Alanyl-LGlutamine, penicillin-streptomycin ( 50 units-50 $\mu \mathrm{g}$ for $\mathrm{ml}$ ) and incubated at $37{ }^{\circ} \mathrm{C}$ in humidified atmosphere of $5 \% \mathrm{CO}_{2}, 95 \%$ air. The culture medium was changed twice a week.

Treatment with antitumour agents and MTT colorimetric assay. Each human cancer cell line (5x103 cells $/ 0.33 \mathrm{~cm} 2)$ were plated in 96 well plates "Nunclon TM Microwell TM" (Nunc) and were incubated at $37^{\circ} \mathrm{C}$. After $24 \mathrm{~h}$, cells were treated with the compounds (final concentration 0.01-100 $\mu \mathrm{M})$. Untreated cells were used as controls. Microplates were incubated at $37{ }^{\circ} \mathrm{C}$ in humidified atmosphere of $5 \% \mathrm{CO}_{2}, 95 \%$ air for 3 days and then cytotoxicity was measured with 
colorimetric assay based on the use of tetrazolium salt MTT (3-(4,5-dimethylthiazol-2-yl)-2,5diphenyl tetrazolium bromide). ${ }^{8}$ The results were read on a multiwell scanning spectrophotometer (Multiscan reader), using a wavelength of $570 \mathrm{~nm}$. Each value was the average of 8 wells (standard deviations were less than 20\%). The $\mathrm{GI}_{50}$ value was calculated according to NCI: thus, $\mathrm{GI}_{50}$ is the concentration of test compound where $100 \mathrm{x}(\mathrm{T}-\mathrm{T} 0) /(\mathrm{C}-\mathrm{T} 0)$ $=50$ ( $\mathrm{T}$ is the optical density of the test well after a 48 -h period of exposure to test drug; $\mathrm{T} 0$ is the optical density at time zero; $\mathrm{C}$ is the control optical density).

\section{Acknowledgements}

Financial support of the University of Catania is gratefully acknowledged.

\section{References}

1. Fortuna, C. G.; Barresi, V.; Berellini, G.; Musumarra, G. Bioorg. Med. Chem. 2008, 16, 4150.

2. Ballistreri, F. P.; Barresi, V.; Benedetti, P.; Caltabiano, G.; Fortuna, C. G.; Longo, M. L.; Musumarra, G. Bioorg. Med. Chem. 2004, 12, 1689.

3. Goodford, P. J. Med. Chem. 1985, 28, 849.

4. Cramer III, R. D.; Patterson, D. E.; Bunce J. J. Am. Chem. Soc. 1988, 110, 5959.

5. Pastor, M.; Cruciani, G.; McLay, I.; Pickett, S.; Clementi, S. J. Med. Chem. 2000, 43, 3233.

6. Barresi, V.; Condorelli, D. F.; Fortuna, C. G.; Musumarra G.; Scirè, S. Bioorg. Med. Chem. 2002, 10, 2899.

7. Ballistreri, F. P.; Barresi, V.; Consiglio, G.; Fortuna, C. G.; Longo, M. L.; Musumarra, G. Arkivoc 2003, part(i), 105.

8. Mosmann, T. J. J. Immunol. Meth. 1983, 65, 55. 